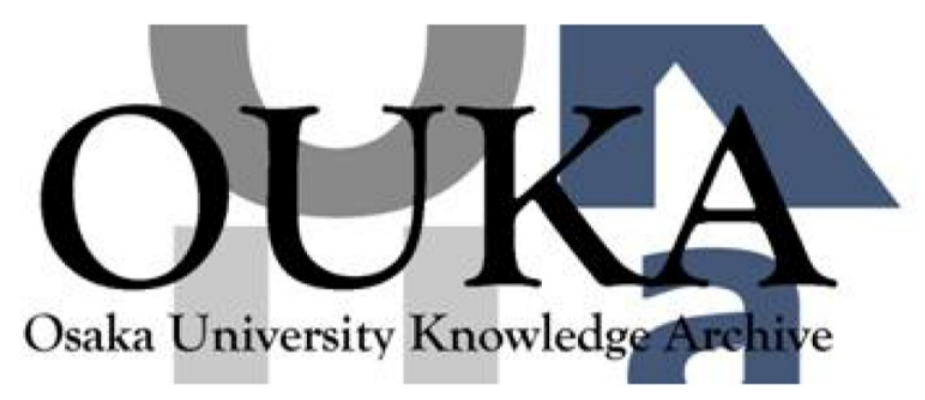

\begin{tabular}{|c|l|}
\hline Title & $\begin{array}{l}\text { Discrete correlation processor as a building } \\
\text { core of a digital optical computing system : } \\
\text { architecture and optoelectronic embodiment }\end{array}$ \\
\hline Author(s) & $\begin{array}{l}\text { Kagawa, Kei ichiro; Ogura, Yusuke; Tanida, Jun } \\
\text { et al. }\end{array}$ \\
\hline Citation & Applied Optics. 38(35) p. 7276-p. 7281 \\
\hline Issue Date & 1999-12-10 \\
\hline oaire:version & VoR \\
\hline URL & https://hdl. handle.net/11094/3240 \\
\hline rights & \\
\hline Note & \\
\hline
\end{tabular}

Osaka University Knowledge Archive : OUKA

https://ir. Library. osaka-u. ac. jp/

Osaka University 


\title{
Discrete correlation processor as a building core of a digital optical computing system: architecture and optoelectronic embodiment
}

\author{
Keiichiro Kagawa, Yusuke Ogura, Jun Tanida, and Yoshiki Ichioka
}

\begin{abstract}
In this paper we present a general-purpose discrete correlation processor (DCP) expected to be the building core block of a digital optical computing system. The DCP-1 is embodied by optoelectronic devices such as a VCSEL and a complementary metal-oxide silicon photodetector. The application targets of the DCP-1 are optical interconnection and various types of digital optical computing. It is expected that digital optical computing techniques coupled with the optoelectronic technology will provide large capability and flexibility in information processing. Introduction of a processing scheme of optical array logic enlarges the applicable field of the DCP-1 as well as its processing capability. With the experimental DCP-1 a bit error rate smaller than $10^{-9}$ was obtained for $A \cdot \bar{B}$ operation under a $500-\mathrm{kHz}$ clock rate. (C) 1999 Optical Society of America

OCIS codes: $200.2610,200.3760,200.4660$.
\end{abstract}

\section{Introduction}

Digital optical computing is a promising technological field, owing to the excellent features of light for information processing, e.g., large parallelism, high-speed, and large communication capability. Smart-pixel technology, ${ }^{1}$ in which integration of semiconductor electronic circuits and optical inputoutput ports is positively applied, is one of the important technical foundations for digital optical computing. Great progress in smart-pixel research enables us to design various kinds of optoelectronic devices with ultrawide communication bandwidths. To show the smart-pixel applications, various demonstration systems have been presented, for example, sorting systems, ${ }^{2}$ optical backplanes, ${ }^{3}$ network routers ${ }^{4}$ and so on. Because of the steady increase in integration density and operation frequency of very large scale integration (VLSI) chips, smart-pixel technology seems to be effective for ultra-high-speed optical interconnection systems.

The authors are with the Department of Material and Life Science, Graduate School of Engineering, Osaka University, 2-1, Yamadaoka, Suita, Osaka 565-0871 Japan. K. Kagawa's e-mail address is Kagawa@mls.eng.osaka-u.ac.jp.

Received 19 February 1999; revised manuscript received 25 June 1999.

0003-6935/99/357276-06\$15.00/0

(C) 1999 Optical Society of America
From the viewpoint of optical application, the current optical interconnection demonstrators use optical properties for simple data links only; thus they do not make the best use of the unique features of light. Therefore there is the question of whether such optical data links will retain their advantage over electronic counterparts when wafer-scale integration and the stacked structure of thinned VLSI chips ${ }^{5}$ become accessible. To explore the utility of the optical technologies, sophisticated applications should be considered.

Motivated by the above findings, we study an advanced form of optical interconnection based on optoelectronic hybrid technologies. The essential idea of the architecture is effective use of optical computing techniques in the optoelectronic hybrid system. Not only simple data transfer but also parallel operations over the image data are achieved by the architecture. The core part of the architecture is a discrete correlating processor (DCP), which is one of the most fundamental operations in digital optical computing.

To provide an example of the DCP, we constructed an experimental processor, DCP-1, with VCSEL's and complementary metal-oxide silicon photodetectors (CMOS-PD's). To our knowledge, the DCP-1 is the first demonstrator of optical-intensive digital optical computing embodied by optoelectronic devices. As a concrete scheme for logic implementation, optical array logic (OAL) is adopted on the DCP-1. With the help of OAL, 6 the system consisting of the DCP-1 can execute a wide range of applications including 


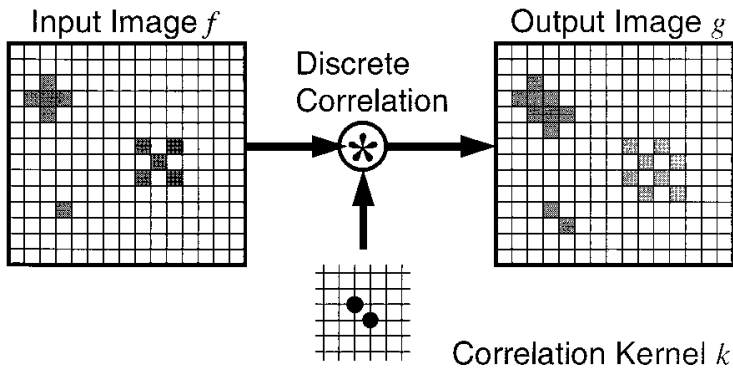

Fig. 1. Fundamental procedure of discrete correlation.

image processing, ${ }^{7-9}$ numerical processing, ${ }^{10}$ database management, ${ }^{11}$ etc.

In this paper, the concept of the DCP is presented and the experimental results obtained by the DCP-1 are described to show the feasibility of the digital optical computing embodied by optoelectronic devices. In Section 2 the architecture of the DCP is explained, and in Section 3 two types of DCP-1 with different configurations are described. In Section 4, experimental results obtained by the experimental DCP-1's are presented and their performance is evaluated.

\section{Architecture}

The fundamental operation performed by the DCP is discrete correlation. Discrete correlation is defined as follows:

$$
g_{i, j}=\sum_{m} \sum_{n} k_{m, n} f_{i-m, j-n}
$$

where $f$ and $g$ are the input and the output images and $k$ is a correlation kernel that determines the contents of the operation. Illustrative explanation of discrete correlation is shown in Fig. 1. Discrete correlation is an essential and commonly used operation in various digital optical computing techniques. As presented by many researchers, discrete correlation coupled with a spatial coding technique enables us to achieve flexible operations on two-dimensional images. ${ }^{12,13}$ In the spatial coding technique a single image or multiple target images are converted into a spatially coded image $f$ according to a predefined coding rule. A logical or arithmetic operation is configured by the contents of the correlation kernel $k$ and the coding rule.

A noncoded technique for optical computing has been presented. ${ }^{14}$ This technique has the potential to achieve optical logic with simple optical components. However, the technique seems difficult to implement practically, owing to a lack of high-speed image display devices and to difficulty in image alignment. On the basis of what we know about current optoelectronic technology, some optoelectronic devices such as VCSEL data should be used for high-speed processing, in which the spatial coding process is not necessarily a troublesome task. In addition, the spatial coding technique can minimize difficulty in alignment, because coded patterns to be overlapped are located neighboring positions. Therefore we adopt the spatial coding scheme in our architecture.

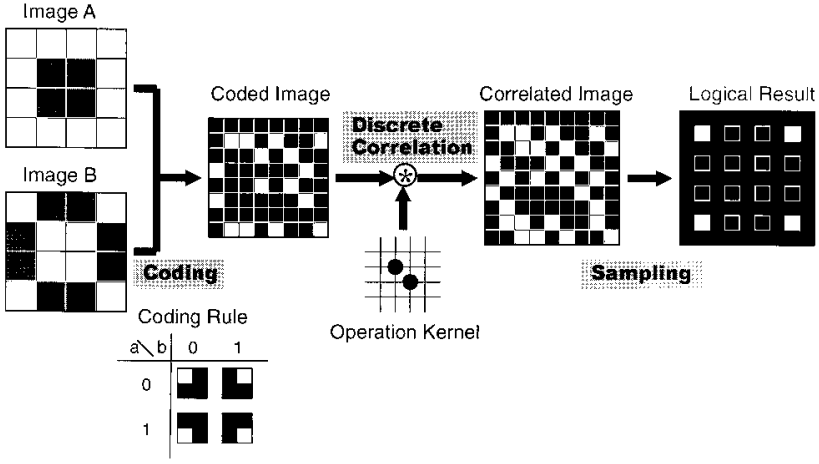

Fig. 2. Procedure of OAL scheme.

As an example of a spatially coded scheme, OAL is explained. Figure 2 shows a schematic diagram of OAL. A pair of binary images are converted into a coded image according to the spatial coding rule. Original images with $N \times N$ pixels are expanded to a coded image with $2 N \times 2 N$ pixels by the encoding. Then discrete correlation is applied to the coded image with an operation kernel, which is identical to the correlation kernel. The pattern of the operation kernel specifies the logical operation. The correlated image is sampled pixel by pixel, and the signal is inverted to obtain the output image. For arbitrary logical operation, oR operation must be executed for a set of sampled images produced by different operation kernels.

Figure 3 shows a block diagram of the DCP architecture. The system is composed of three blocks: image emitter, optical correlator, and image detector. For the image emitter an array of point sources, e.g., a VCSEL array, is used. The optical correlator performs discrete correlation with various optical setups. As the image detector, a high-speed semiconductor array sensor can be used. Since all the operations in the DCP are executed in parallel, the degree of parallelism directly affects the processing performance. Therefore scalability of the performance in space and time is an important feature of the architecture.

The DCP can be applied to various application fields: simple optical interconnection, parallel processing based on a spatial coding technique, and

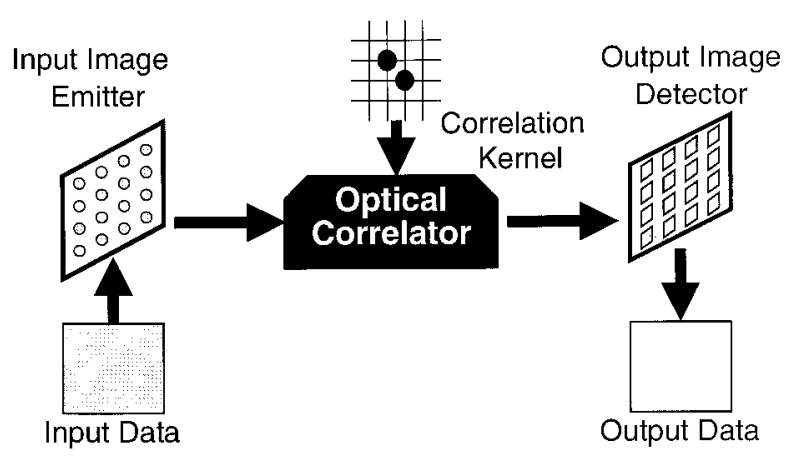

Fig. 3. Architecture of DCP. 


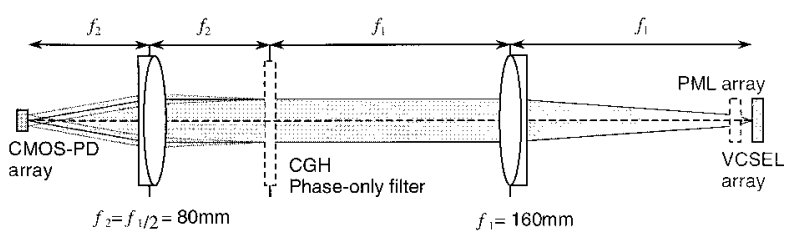

(a)

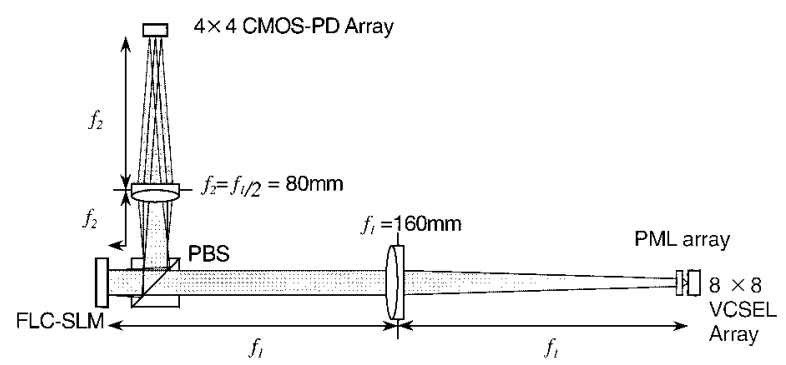

(b)

Fig. 4. Optical setups for the DCP-1 of (a) type A and (b) type B.

computing-oriented optical interconnection. ${ }^{15,16}$ Centering a core processor based on the DCP, we can construct an optoelectronic hybrid computing system effectively.

\section{DCP-1: Optoelectronic Embodiment}

As examples of the DCP, two experimental systems were constructed. One is a static kernel system (type A), and the other is a dynamic kernel system (type B). Figure 4 shows their optical setups. As the image emitter, an $8 \times 8$ VCSEL array (Gigalase, Micro Optical Devices; emitting wavelength, $850 \mathrm{~nm}$; pixel pitch, $250 \mu \mathrm{m}$ ) was used. The VCSEL's were driven by the driver supplied by the United States-Japan Joint Optoelectronics Project (JOP), whose maximum operation speed is estimated by SPICE simulation to be greater than $100 \mathrm{MHz}$. The swing of the driving current is controlled by bias voltage $V_{\text {bias }}$ and modulation voltage $V_{\mathrm{ss}}$. For the optical correlator, a 4- $f$ Fourier transform configuration was adopted with a computergenerated hologram (CGH) filter specifying the corre- lation kernel. The correlated image was detected by a CMOS-PD array with $4 \times 4$ pixels (supplied by JOP). The maximum operation speed of the detector is 15 $\mathrm{MHz}$ for $100 \mu \mathrm{W}$ of incident light. The pixel pitch and the photosensitive areas are $250 \mu \mathrm{m} \times 250 \mu \mathrm{m}$ and $110 \mu \mathrm{m} \times 110 \mu \mathrm{m}$, respectively. The photosignal is amplified and binarized by a series of two comparators in the receiver circuit. The threshold signal level can be controlled by two external voltages, $V_{t 1}$ and $V_{t 2}$, which are threshold voltages to the first and the second comparators. Because the pixel pitches of the VCSEL array and the CMOS-PD array are identical, the magnification ratio of the optical system $f_{2} / f_{1}$ must be set as 0.5 to satisfy the sampling condition of the OAL scheme.

The difference between two types of DCP- 1 is the method of $\mathrm{CGH}$ embodiment. Although the performance of recent spatial light modulators (SLM's) has increased notably, there is no candidate for the CGH filter that can control the wave front of light signals at the high speed rate with high optical efficiency and contrast ratio. As an alternative solution, two kinds of prototype were considered for the different application targets. In general, static filters can be realized by optical passive devices, which provide fast operation speed in exchange for processing flexibility. Dynamic filters are attractive for flexibility and applicability. However, their optical transmission is relatively low, and the setup time for changing the filter patterns is too slow compared with the transmission bit rate of the optical signals. The optical component devices used in both types of DCP-1 are summarized in Table 1 . The lenses in the optical systems are common for convenience of changing the configurations.

The CGH filter for the type A system is embodied by a binary phase-only filter etched on a $\mathrm{SiO}_{2}$ substrate. The filter pattern is exposed by a laser beam writer ${ }^{17}$ onto photoresist (Model AZ1500, Hoechst Industry Ltd.) spin-coated on a $\mathrm{SiO}_{2}$ substrate. After the development the pattern is etched by ionized $\mathrm{CF}_{4}$ gas. In the type B system the filter pattern is directly displayed on a ferroelectric liquid crystal(FLC-) SLM (SLM Developer Kit, Displaytech) with binary amplitude modulation. The resolution and

Table 1. Specifications of Optoelectronic Devices Used in the DCP-1

\begin{tabular}{|c|c|c|c|c|c|c|}
\hline Product & Supplier & Array Size & $\begin{array}{c}\text { Pixel } \\
\text { Pitch }(\mu \mathrm{m})\end{array}$ & $\begin{array}{l}\text { Pixel Size } \\
\quad(\mu \mathrm{m})\end{array}$ & $\begin{array}{l}\text { Operational } \\
\text { Speed }\end{array}$ & Etc. \\
\hline $\begin{array}{l}\text { VCSEL array, } \\
\text { Gigalase }\end{array}$ & Micro Optical Devices & $8 \times 8$ & 250 & $\phi 8$ & - & - \\
\hline VCSEL driver & JOP & $16 \mathrm{I} / \mathrm{O}^{a}$ & - & - & $>100 \mathrm{MHz}$ & - \\
\hline $\begin{array}{l}\text { Ferroelectric spatial } \\
\text { light modulator, } \\
\text { SLM Developer } \\
\text { Kit }\end{array}$ & Displaytech & $256 \times 256$ & 15 & 14 & $\begin{array}{c}2.5 \mathrm{kHz} \text { at } \\
25^{\circ} \mathrm{C}\end{array}$ & $\begin{array}{c}\text { Reflectivity, } \\
<20 \% \text {; peak oper- } \\
\text { ating wavelength, } \\
680 \mathrm{~nm}\end{array}$ \\
\hline CMOS photodetector & JOP & $4 \times 4$ & 250 & 110 & $15 \mathrm{MHz}$ & - \\
\hline $\begin{array}{l}\text { Planar microlens } \\
\text { array }^{b}\end{array}$ & Micro-Opt & $\begin{array}{r}0.96 \mathrm{~mm} \\
\times 0.96 \mathrm{~mm}\end{array}$ & 250 & $\phi 250$ & - & $\begin{array}{c}\text { Focal length, } 720 \\
\mu \mathrm{m} \text { at } 850 \mathrm{~nm}\end{array}$ \\
\hline
\end{tabular}


the pixel pitch of the FLC-SLM are $256 \times 256$ and 15 $\mu \mathrm{m}$, respectively. The contrast ratio of the displayed image is 100:1 (zero order at $633 \mathrm{~nm}$ ), and the maximum operation speed is $2.5 \mathrm{kHz}$ at $25{ }^{\circ} \mathrm{C}$.

A practical problem of the VCSEL array is its large divergence angle, typically $\pm 15^{\circ}$, which causes optical loss, owing to the small aperture size of the lenses and the SLM. To converge the beam into the aperture of the FLC-SLM, 3.84- $\mathrm{mm}^{2}$, we use a planar microlens (PML) array (Model PML-FW0250S0096SNC, Micro-Opt). The pitch of the lenslets is identical to that of the VCSEL array $(250 \mu \mathrm{m})$, and the focal length is $720 \mu \mathrm{m}$ at $850 \mathrm{~nm}$.

To design the CGH filters, we used a method based on the Gerchberg-Saxton algorithm. ${ }^{18,19}$ The basic procedure of the algorithm is iteration of a sequence of Fourier and inverse-Fourier transformations under constraints to obtain the target intensity profile of the point-spread function. In our design the following parameters were used: the pixel pitch of VCSEL array $\delta$, the number of VCSEL's $N \times N$, the pixel pitch of the filter $\Delta$, the wavelength of the VCSEL's $\lambda$, and the focal length of the second lens $f_{2}$ shown in Fig. 4 . Figure 5 shows the reconstructed image of the CGH filter, which is identical to the pattern of the correlation kernel. When an image is displayed at the VCSEL array, its correlated image is obtained at the position of the -1 st diffraction. For full interconnection between all pixels in the input image the size of the correlation kernel is $2 N-1$. The minimum value of $f_{2}$ is given by the condition that the 0th diffraction image of the VCSEL array and the correlated image by the -1 st diffraction do not overlap. Considering the parameters $\delta=250 \mu \mathrm{m}, \lambda=850 \mathrm{~nm}, \Delta=15 \mu \mathrm{m}$ (SLM), and $N=8$, we determine that $f_{2}$ is $80 \mathrm{~mm}$; so the margin between the two images is $133.3 \mu \mathrm{m}$.

\section{Experimental Results}

To evaluate the performance of the constructed DCP-1's, we observed several characteristics including the optical efficiency of the CGH filter, the maximum frequency of electric and optical signals, and the speed of logical operation.

Figure 6 shows the electric and the optical signals observed in the experimental system. The waveforms of the function generator (Model 33120A, Hewlett Packard), the VCSEL driver, and the optical output detected by the avalanche photodetector module (Model S5331-01; Hamamatu Photonics; bandwidth $100 \mathrm{MHz}$ ) are depicted for several operation speeds. For the VCSEL driver, $V_{\text {bias }}$ and $V_{\text {ss }}$ were set to 4.95 and $6.1 \mathrm{~V}$, respectively. The optical intensity was $22 \mu \mathrm{W}$ (measured by an optical power meter), and the contrast ratio between the on and the OFF states of the VCSEL was more than 100:1. As seen from the results, the driver circuit functions at least up to $8 \mathrm{MHz}$.

Optical efficiency is an important factor for determining the operation frequency of the optoelectronic system. Table 2 shows the measured optical efficiencies to verify the effect of the PML array. With the PML array the divergence angle of the emitting

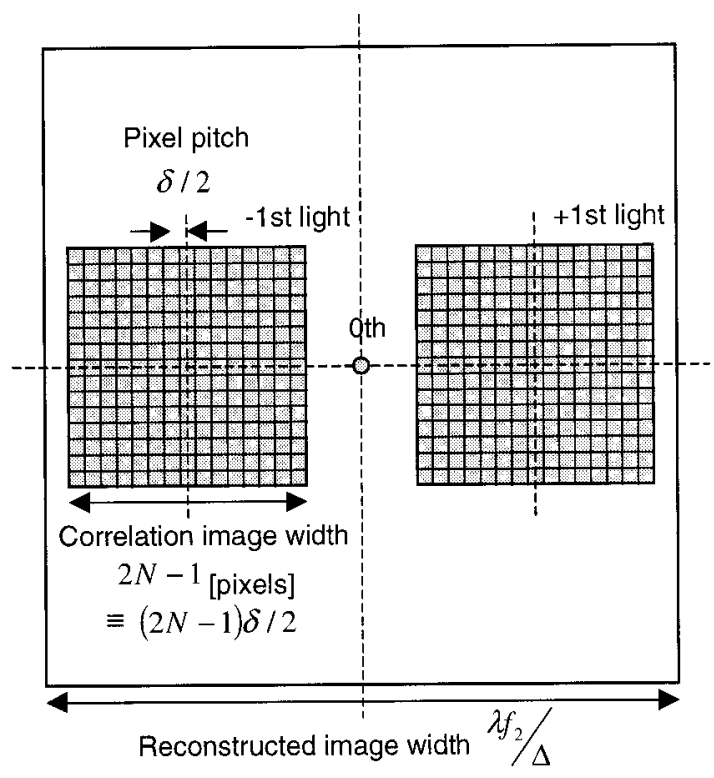

(a)

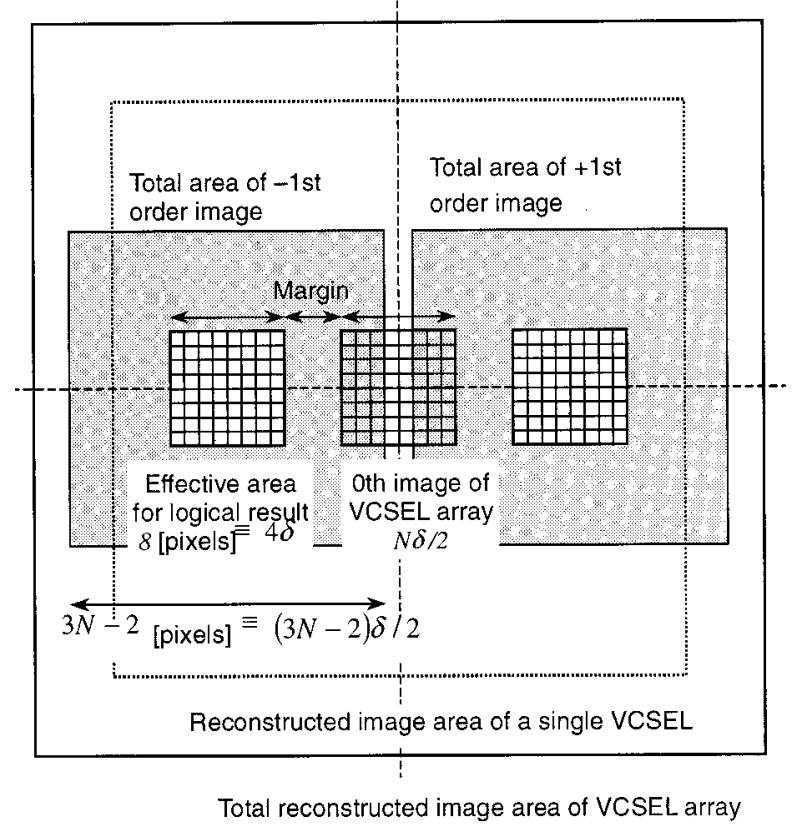

(b)

Fig. 5. Reconstructed image plane mapping for (a) a single light source and (b) a VCSEL array.

beam from the VCSEL is reduced to $\pm 0.9^{\circ}$ from $\pm 15^{\circ}$ so that the optical efficiency is improved to be $13.75 \%$ (4.3 times the case without the PML array). The main reason for the remaining optical loss is the mismatch between the divergence angle of the VCSEL and the field angle of the PML.

We reconstructed several CGH filters in the type A and type B systems to observe the diffraction efficiency and the quality of the output images. We designed a CGH filter to execute an operation $\overline{A \oplus B}$, whose fan-out number is 2 . The pixel size and the pattern size were the same in both systems, which 


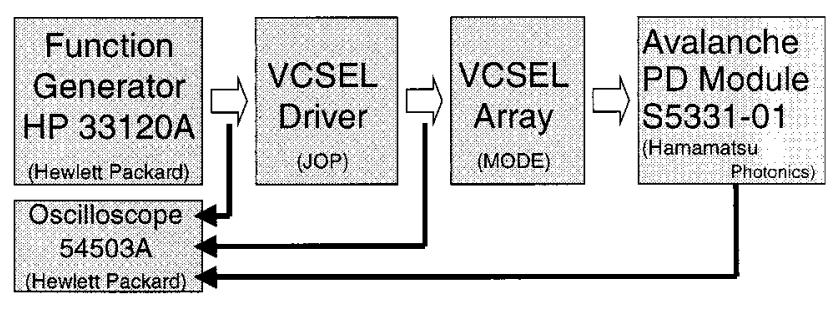

(a)

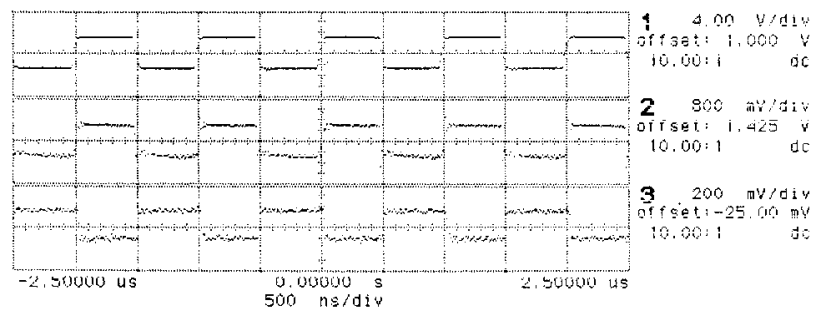

(b) $1 \mathrm{MHz}$

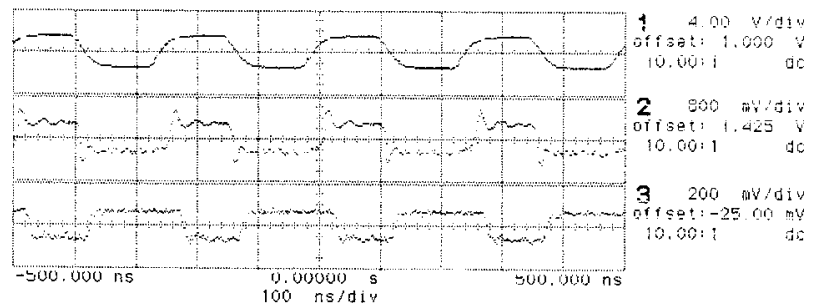

(c) $4 \mathrm{MHz}$

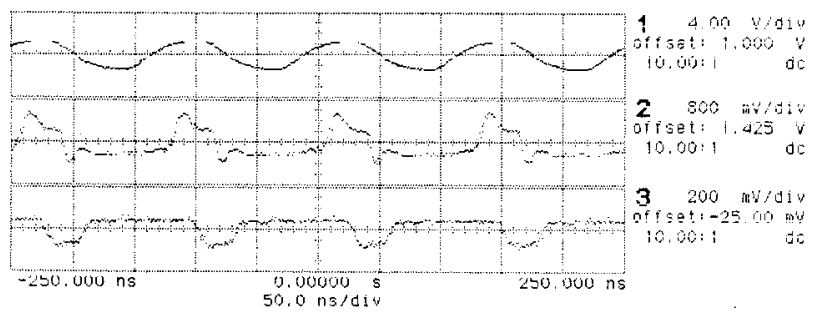

(d) $8 \mathrm{MHz}$

Fig. 6. (a) System block diagram for measurement of signals. (b)-(d) Measured waveforms; $\mathrm{CH} 1$ and $\mathrm{CH} 2$ are output signals of a function generator (Model 33120A, Hewlett Packard) and a VCSEL driver, respectively; CH3 is amplified photocurrent from an avalanche photodiode module (Model S5331-01, Hamamatu Photonics). were $15 \mu \mathrm{m} \times 15 \mu \mathrm{m}$ and $256 \times 256$ pixels, respectively. Observed diffraction efficiencies of the CGH filters were $37.14 \%$ (type A) and $5.2 \%$ (type B). The reason for low diffraction efficiency of type $B$ is that the SLM is operated by amplitude modulation. Figure 7 shows experimental results of the correlation. The average diameters of the type $\mathrm{A}$ and type $\mathrm{B}$ reconstructed spots were 60 and $115 \mu \mathrm{m}$, which were smaller than the detector area, $120 \mu \mathrm{m} \times 120 \mu \mathrm{m}$. The differences between the theoretical and the experimental correlation patterns in Fig. 7 were caused by a malfunction of the VCSEL drivers.

On the type A system we measured bit error rate (BER) of operations $A \cdot \bar{B}$ and $\overline{A \oplus B}$ for pseudorandom sequences of input signals. The BER is defined as the ratio of the number of logical errors to the total number of pairs of input bit signals. Since operation $A \cdot \bar{B}$ is achieved by an operation kernel with a single point pattern, we can implement it by a simple imaging system with lateral translation so that no CGH filter is inserted and so that the system shows the maximum performance. In the measurement the $10^{9}$-bit-long pseudorandom sequences were generated by a personal computer (PC), and the optical outputs were stored in the memory of the PC to calculate the BER. On the experimental system no error occurred in the measurement of logical operation $A \cdot \bar{B}$ under $500 \mathrm{kHz}$, which was restricted by the maximum speed of the PC's input-output. Therefore we concluded the BER was smaller than $10^{-9}$ under $500 \mathrm{kHz}$. For the operation $\overline{A \oplus B}$ the BER was $10^{-5}$ at $25 \mathrm{kHz}$ for the $10^{5}$-bit-long pseudorandom sequence. Degradation of the contrast ratio of the correlation image and the optical efficiency of the system forced us to reduce the operation speed. In the type B system the CMOS-PD could not detect the logical results, because the light intensity was too weak for the CMOS photodetectors (but was sufficient for the CCD camera for observing the correlation images). The reasons for detection failure are assumed to be the mismatch between the designed specification of the peak operating wavelength of the

Table 2. Optical Efficiencies for the Different Setups ${ }^{a}$

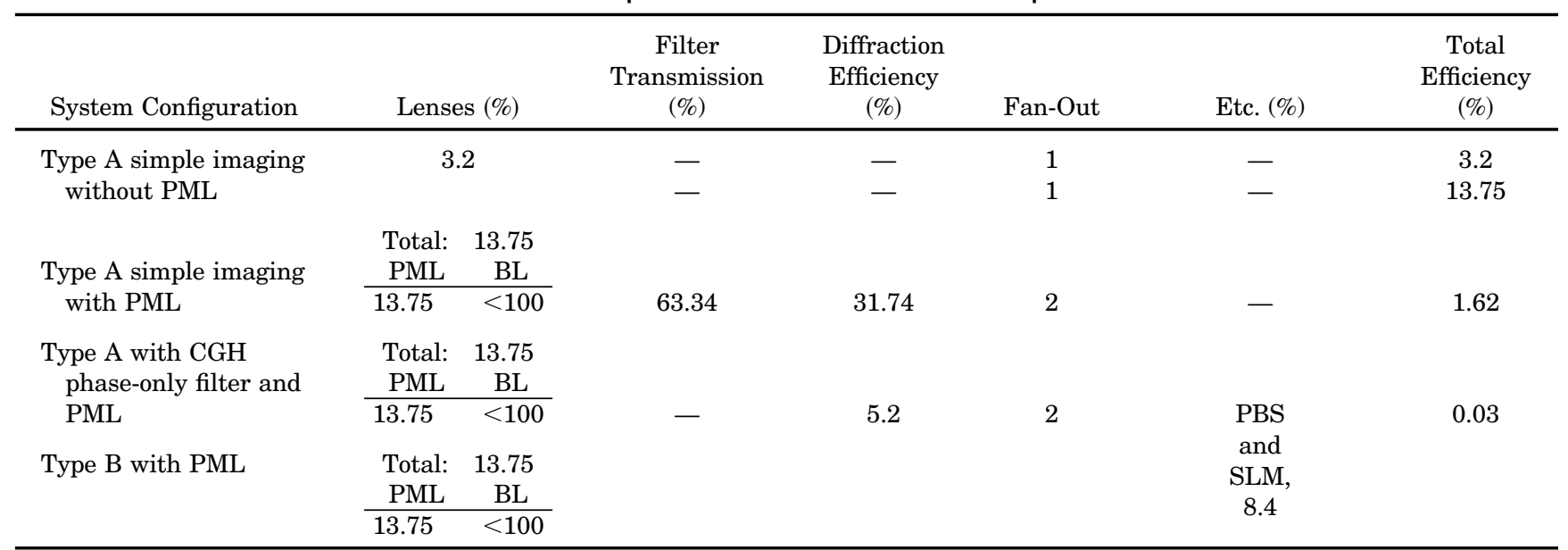

${ }^{a} \mathrm{BL}$, balk lens; PBS, polarized beam splitter. 


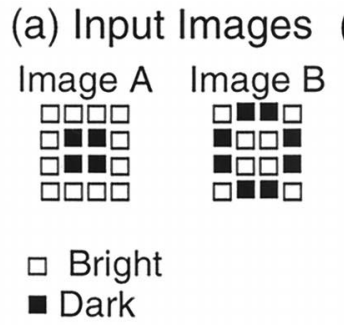

(b)

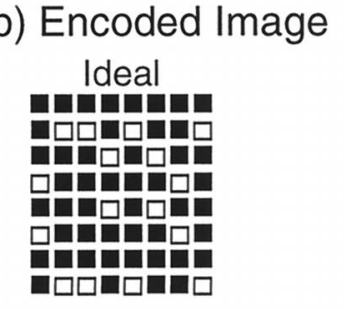

(c) Correlated Image

Ideal
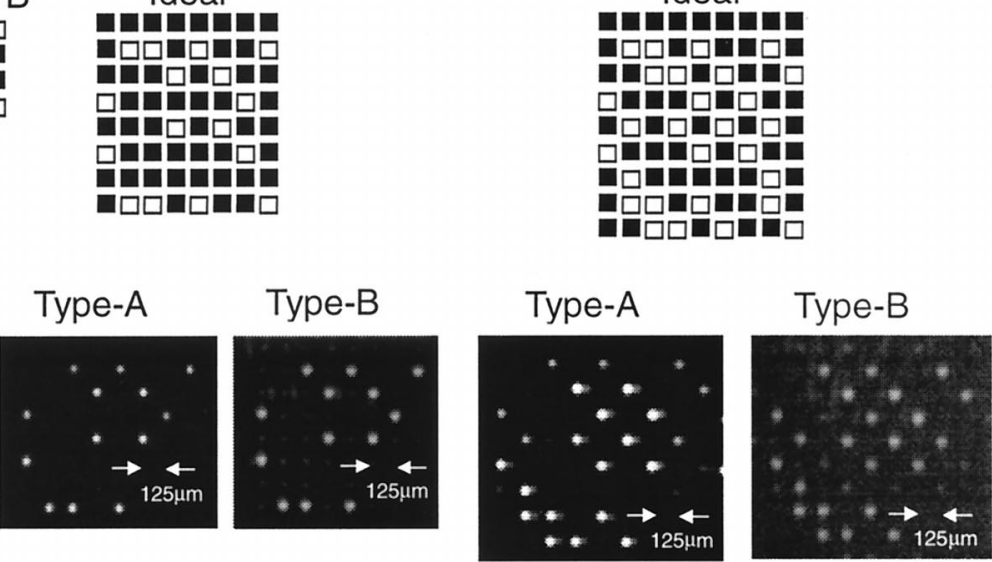

Fig. 7. Encoded and correlated images of types A and B for a pair of input images.

SLM, $680 \mathrm{~nm}$, and the emission wavelength of the VCSEL's, $850 \mathrm{~nm}$, as well as the low diffraction efficiency of the SLM caused by amplitude modulation.

\section{Conclusions}

In this paper we have presented a discrete correlation processor (DCP) as a building core block of a digital optical computing system. On the basis of state-ofthe-art optoelectronic technologies, two types of system (DCP-1) were constructed. We adopted an OAL scheme on the DCP-1 and successfully confirmed the achievement of optical logic operations. For the DCP-1 with a static kernel, the experimental results show that the maximum frequency of the electric circuit is $8 \mathrm{MHz}$ and that the BER is smaller than $10^{-9}$ for a logical operation at $500 \mathrm{kHz}$. The maximum speed is restricted by the response time of the photodetector. Because the response time is heavily affected by the incident optical power, improvement of the optical power efficiency is a critical issue for future development.

This research was supported by JOP user funding from the Real World Computing Partnership (RWCP). The authors are grateful for the activities of JOP.

\section{References}

1. T. Kurokawa, S. Matso, T. Nakahara, K. Tateno, Y. Ohiso, A. Wwakatsuki, and H. Tsuda, "Design approaches for VCSEL's and VCSEL-based smart pixels toward parallel optoelectronic processing systems," Appl. Opt. 37, 194-204 (1998).

2. D. T. Neilson, S. M. Prince, D. A. Baillie, and F. A. P. Tooley, "Optical design of a 1024-channel free-space sorting demonstrator," Appl. Opt. 36, 9243-9252 (1997).

3. D. V. Plant, "Constructing a free space optical backplane: challenges and choices," in Optics in Computing, Vol. 8 of 1997 OSA Technical Digest Series (Optical Society of America, Washington, D.C., 1997), pp. 156-158.

4. T. Mark Pinkston, M. Raksapatchawarong, and Y. Choi, "WARRP core: optoelectronic implementation of networkrouter deadlock-handling mechanisms," Appl. Opt. 37, 276283 (1998).

5. M. Koyanagi, H. Kurino, K. Sakuma, and K. W. Lee, "Future system-on-silicon LSI systems," IEEE Mirco. 18, 17-22 (1998).
6. Y. Ichioka and J. Tanida, "Optical parallel logic gates using a shadow-casting system for optical digital computing," Proc. IEEE 72, 787-801 (1984).

J. Tanida, T. Konishi, and Y. Ichioka, "P-OPALS: pure optical parallel array logic system,” Proc. IEEE 82, 1668-1677 (1994).

7. J. Tanida and Y. Ichioka, "Programming of optical array logic. 1. Image data processing," Appl. Opt. 27, 29262930 (1988).

8. J. Tanida and Y. Ichioka, "Programming of optical array logic. 2. Image data processing," Appl. Opt. 27, 2931-2939 (1988).

9. S. Kakizaki, J. Tanida, and Y. Ichioka, "Gray image processing using optical array logic,” Appl. Opt. 31, 1093-1102 (1992).

10. J. Tanida, M. Fukui, and Y. Ichioka, "Programming of optical array logic. 2. Numerical data processing based on pattern logic," Appl. Opt. 27, 2931-2939 (1988).

11. M. Iwata, J. Tanida, and Y. Ichioka, "Database management using optical array logic,” Appl. Opt. 32, 1987-1995 (1993).

12. K. H. Brenner, A. Huang, and N. Streibl, "Digital optical computing with symbolic substitution," Appl. Opt. 25, 3054-3060 (1986).

13. M. Fukui and K. Kitayama, "Applications of image logic algebra: wire routing and numerical data processings," Appl. Opt. 31, 4645-4656 (1992).

14. S. Jutamulia and G. Storti, "Noncoded shadow-casting logic array," Appl. Opt. 28, 4517-4518 (1989).

15. Y. Awatsuji, N. Sakamotom, H. Utsuro, J. Tanida, and Y. Ichioka, "Optical array logic network computing: design of a prototype system," Opt. Rev. 4, 199-202 (1997).

16. Y. Awatsuji, N. Sakamotom, J. Tanida, and Y. Ichioka, "Design and construction of experimental optical array logic network computing system," in Optics in Computing, Vol. 8 of 1997 OSA Technical Digest Series (Optical Society of America, Washington, D.C., 1997), pp. 9-11.

17. Y. Ogura, Y. Ichioka, S. Taniguchi, Y. Mokuno, and K. Matsuoka, "Design of phase only optical element for laser beam drawing system with high resolution," in Extended Abstracts of Optics Japan (Optical Society of Japan, Tokyo, 1998), pp. 47-48 (in Japanese).

18. R. W. Gerchberg and W. O. Saxton, "A practical algorithm for the determination of phase from image and diffraction plane pictures," Optik (Stuttgart) 35, 237-246 (1972).

19. F. Wyrowski, "Diffractive optical elements: iterative calculation of quantized, blazed phase structures," J. Opt. Soc Am. A 7, 961-969 (1990). 